\title{
Non-invasive evaluation of ventricular refractoriness and its dispersion during ventricular fibrillation in patients with implantable cardioverter defibrillator
}

\author{
Jijian Luo, Carl Magnus Pripp, Eva Hertervig, Ole Kongstad, Erik Ljungström, \\ S Bertil Olsson and Shiwen Yuan*
}

Address: Department of Cardiology, University Hospital, Lund University, Lund, Sweden

Email: Jijian Luo - Jijian.Luo@kard.lu.se; Carl Magnus Pripp - Carlmagnus.Pripp@kard.lu.se; Eva Hertervig - Eva.Hertervig@kard.lu.se; Ole Kongstad - Ole.Kongstad@kard.lu.se; Erik Ljungström - Erik.Ljungstrom@kard.lu.se; S Bertil Olsson - Bertil.Olsson@kard.lu.se;

Shiwen Yuan* - Shiwen.Yuan@kard.lu.se

* Corresponding author

Published: 09 June 2004

BMC Cardiovascular Disorders 2004, 4:8

This article is available from: http://www.biomedcentral.com/|47|-226I/4/8

(c) 2004 Luo et al; licensee BioMed Central Ltd. This is an Open Access article: verbatim copying and redistribution of this article are permitted in all media for any purpose, provided this notice is preserved along with the article's original URL.
Received: 04 February 2004

Accepted: 09 June 2004

\begin{abstract}
Background: Local ventricular refractoriness and its dispersion during ventricular fibrillation (VF) have not been well evaluated, due to methodological difficulties.

Methods: In this study, a non-invasive method was used in evaluation of local ventricular refractoriness and its dispersion during induced VF in II patients with VF and/or polymorphic ventricular tachycardia (VT) who have implanted an implantable cardioverter defibrillator (ICD). Bipolar electrograms were simultaneously recorded from the lower oesophagus behind the posterior left ventricle (LV) via an oesophageal electrode and from the right ventricular (RV) apex via telemetry from the implanted ICD. VF intervals were used as an estimate of the ventricular effective refractory period (VERP). In 6 patients, VERP was also measured during sinus rhythm at the RV apex and outflow tract (RVOT) using conventional extra stimulus technique.
\end{abstract}

Results: Electrograms recorded from the RV apex and the lower esophagus behind the posterior LV manifested distinct differences of the local ventricular activities. The estimated VERPs during induced VF in the RV apex were significantly shorter than that measured during sinus rhythm using extra stimulus technique. The maximal dispersion of the estimated VERPs during induced VF between the RV apex and posterior LV was that of 10 percentile VF interval (40 $\pm 27 \mathrm{~ms})$, that is markedly greater than the previously reported dispersion of ventricular repolarization without malignant ventricular arrhythmias $(30-36 \mathrm{~ms})$.

Conclusions: This study verified the feasibility of recording local ventricular activities via oesophageal electrode and via telemetry from an implanted ICD and the usefulness of VF intervals obtained using this non-invasive technique in evaluation of the dispersion of refractoriness in patients with ICD implantation.

\section{Background}

Sudden cardiac death due to ventricular tachyarrhythmias is among the most devastating manifestations of heart disease, which remains a major unresolved public health 
problem [1]. Inhomogeneity in ventricular refractoriness and/or repolarization has been widely recognized as an important factor underlying the initiation of ventricular tachycardia (VT) and ventricular fibrillation (VF) $[2,3]$. It has been assumed that during multiple wavelet reentry, cells are re-excited as soon as they have regained their excitability [4,5]. Some previous studies [6-9] have reported that the local VF interval could be an index of ventricular effective refractory periods (VERP). However, local refractoriness of ventricle and its dispersion in human hearts has not been well evaluated, due to methodological difficulties. In the present study, we used a noninvasive method to simultaneously record local electrical activities from the left and right ventricles. The aim was to investigate the usefulness of this method in evaluating the local refractoriness and its dispersion between the right and left ventricles during episodes of induced VF in patients with an implantable cardioverter defibrillator (ICD).

\section{Methods \\ Patients}

Eleven patients with VF or polymorphic VT who have implanted an ICD were studied. They were 10 males and 1 female with mean $60.3 \pm 13.4$ years (range 30 to 76 years). All patients have indication for implantation of an ICD device. All antiarrhythmic drugs had been withdrawn for a period equivalent to at least five half-lives before the study. No patients had amiodarone treatment within the previous three months. None of the patients had electrolyte abnormalities.

Diagnosis was verified by evaluation of medical history, ECG documentation of VF and/or polymorphic VT and induction of these arrhythmias during an electrophysiological study, prior to ICD implantation. Coronary heart disease was present in 8 patients (4 of them undergone coronary artery bypass grafting and 2 undergone percutaneous transluminal coronary angioplasty), non-structural heart disease in 3 patients. The presenting arrhythmias were primary VF in 6 patients, polymorphic VT in 5 patients that soon degenerated into VF.

The study protocol was approved by the local ethical committee and confirmed with the principles outlined in the Declaration of Helsinki, and the electrophysiological procedures were in accordance with local institutional guidelines. Informed consent was obtained from all patients.

\section{Electrophysiological protocols}

The study was conducted in combination with a postimplantation control study with 1 week after the implantation of an ICD. In brief, a bipolar trans-oesophageal electrode catheter (interpolar distance $10 \mathrm{~mm}$ ) was positioned in the lower oesophagus near the cardia behind the left ventricle (LV), as verified by fluoroscopy and electrograms, for recording electrograms from the posterior LV. The electrogram with predominant ventricular electrograms of distinct and rapid deflections was used as sign that the electrode was in the lower part of oesophagus behind the posterior LV. The endocardial ICD electrodes at the right ventricular (RV) apex (interpolar distance 9 $\mathrm{mm}$ ) were used for recording bipolar electrograms from the RV via telemetry. Bipolar electrograms of both the right and left ventricles were simultaneously recorded.

In 6 of the 11 patients, the VERP was also measured via the ICD electrode at the RV apex during sinus rhythm before the VF induction. In 6 patients, the VERP was also measured at the RV apex and outflow tract (RVOT) using a drive-train cycle length $\left(S_{1}\right)$ of 400 and $600 \mathrm{~ms}$ during a previous electrophysiological study.

The VF was induced by high rate burst pacing at the RV apex via the ICD electrode. VF was defined as ventricular arrhythmia showing irregular waves of variable morphology and amplitude in surface ECG, with no identifiable QRS complexes and baseline, the rate is between 300 to 500 beat $/ \mathrm{min}$. The induced VF episodes were successfully terminated by the ICD in all patients.

Bipolar electrograms were recorded using a multi-channel electrophysiological recorder (Bard electrophysiology Lab System) at a filter bandwidth of 50-500 Hz. Twelve-lead surface ECG was also simultaneously recorded.

\section{Signal analysis}

Bipolar electrogram recordings from the RV apex and posterior LV during induced VF were used. The local VF intervals were manually measured on printed papers or on screen using a custom-made program with manual corrections. When the local activation was difficult to define due to low amplitude and/or fragmented, continuous local activities, the intervals before and after were omitted from analysis to ensure an accurate measurement of the VF interval.

The mean, medium, 10th percentile and the shortest VF intervals of both the RV apex and posterior LV were calculated as estimates of local VERPs during VF. The dispersion of the mean, medium, 10th percentile and the shortest VF intervals were defined as the difference of the mean, medium, 10th percentile and the shortest VF intervals between the RV apex and posterior LV. The mean variance of VF intervals was calculated from the variances of all VF episodes at the RV apex and posterior LV. The differences of the estimated VERPs and the variances between the two sites were used as measurements of the dispersion of ventricular refractoriness during induced VF. 


\section{Statistical analysis}

All data were expressed as mean \pm standard deviation in millisecond. Student's t test for unpaired and paired samples was used to assess the significance of the difference in parameters between the two ventricles. A value of $\mathrm{p}<0.05$ was considered as statistically significant.

\section{Results}

Thirty-four episodes of VF were induced, lasted from 8.2 to 14.2 seconds, in the 11 patients. Mean duration of the induced VF episodes was $10.3 \pm 2.0$ seconds. No complications were observed. During the episodes of the induced $\mathrm{VF}$, electrograms from the endocardium at the RV apex and the lower esophagus behind the posterior LV manifested distinct local ventricular activities (Figure 1).

To estimate VERP during the induced VF, a mean of $61 \pm$ $16 \mathrm{VF}$ intervals were measured in the 11 patients. An average of 2 pieces of recordings (ranged 1-4) was omitted from analysis due to low amplitude, fragmented and/or continuous electrical activities, where the local activation was difficult to define.

The estimated VERP parameters at the RV apex and posterior LV were shown in Table 1 . The dispersion of the 10th percentile and the shortest VF intervals were approximately threefold of that of the mean and medium VF intervals. The maximal dispersion of the estimated VERPs was that of the 10 th percentile interval, which was $40 \pm 27$ ms. The difference of the variances between the RV apex and posterior LV, i.e. the dispersion of the variance, was $620 \pm 595$.

The VERPs measured in 6 patients during sinus rhythm using extra-stimulus technique were $222 \pm 21 \mathrm{~ms}$ and 223 $\pm 22 \mathrm{~ms}$ in the RV apex and RVOT respectively at the basic cycle lengths of $400 \mathrm{~ms}$, and $241 \pm 26 \mathrm{~ms}$ and $240 \pm 16 \mathrm{~ms}$ at the basic cycle length of $600 \mathrm{~ms}$. The estimated VERPs during the induced VF were significantly shorter than that measured at the same site during sinus rhythm using extra-stimulus technique (all $\mathrm{p}<0.05$ ).

\section{Discussion}

The main findings of this study are as follows: (a) it is possible to non-invasively record ventricular electrical activities during VF simultaneously from the RV apex via telemetry from an implanted ICD and from the posterior LV via an esophagus electrode. (b) Distinctly different activities from the two sites in the right and left ventricles suggest the potential usefulness of this method in evaluation of the local ventricular refractoriness and its dispersion in the patient during episodes of induced VF. (c) Our observation in a limited number of patients showed that the dispersion of refractoriness between the RV apex and the posterior LV is markedly larger than the previously reported dispersion of repolarization in patients without malignant ventricular arrhythmias $[14,15]$.

The VERP is conventionally measured during sinus rhythm using extra-stimulus technique. However, the VERP during VF could not be tested using this technique. Fibrillation intervals have been used as an index of local refractoriness and shown to correlate well with the local atrial and ventricular refractoriness determined using extrastimulus technique in animal [4,6-8] and human subjects [5,9] in serval previous studies. This method is based on the assumption that during fibrillation, cells are reexcited by one of many multiple wavelet as soon as their refractoriness ends and recovers their excitablity. In animal models and in patients undergoing open chest surgry, VF interval has been used as an index of local ventricular refractoriness. Ramdat Misier et al [9] measured local VF intervals using multiple channels of epicardial eletrogram in patients with VT or VF after myocardial infarction. They found that the dispersion of refractory periods in the myocardium remote from the infarction was larger in patients with postinfarction VF than in patients with postinfarction VT. However, this method can only be applied in patients undergoing opened chest surgery during cardiopulmonary bypass.

Little is know about the spatial dispersion of refractoriness between the RV and LV in the human hearts, due to ethical limitations and difficulties in recording local ventricular electrical activities simultaneouslyfrom the right and left ventricles. Franz et al [14] recorded endocardial monophasic actional potentials from 8 sites in the right and 8 sites in the left ventricles in a patient with concordant $\mathrm{T}$ waves and found the maximal dispersion of repolarization time among these sites was $36 \mathrm{~ms}$. Similarly, Shimizu et al [15] showed the dispersion of monophasic actional potential duration among 2 sites in the right and one site in the left ventricles was $30 \mathrm{~ms}$ in a patient with sick sinus syndrome, and in their six patients with congenital long QT syndrome the dispersion was $30 \pm 5 \mathrm{~ms}$. To our knowledge, data of the dispersion of ventricular refractoriness between the RV and LV in patients with malignant ventricular arrhythmias other than congenital long QT syndrome are still lacking.

In the present study, we simultaneously recorded bipolar electrograms from the RV apex via telemetry from an implanted ICD and from electrode in the lower esophagus behind the posterior LV. It is clear that the esophageal electrodes had no direct contact with the the left ventricular myocardium and the reported distance between the electrods is in average $20 \mathrm{~mm}$, which would be shorter immediately below the cardia [10-12]. However, distinctly different signals of ventricular electrical activities at the RV apex and posterior LV during induced VF were 


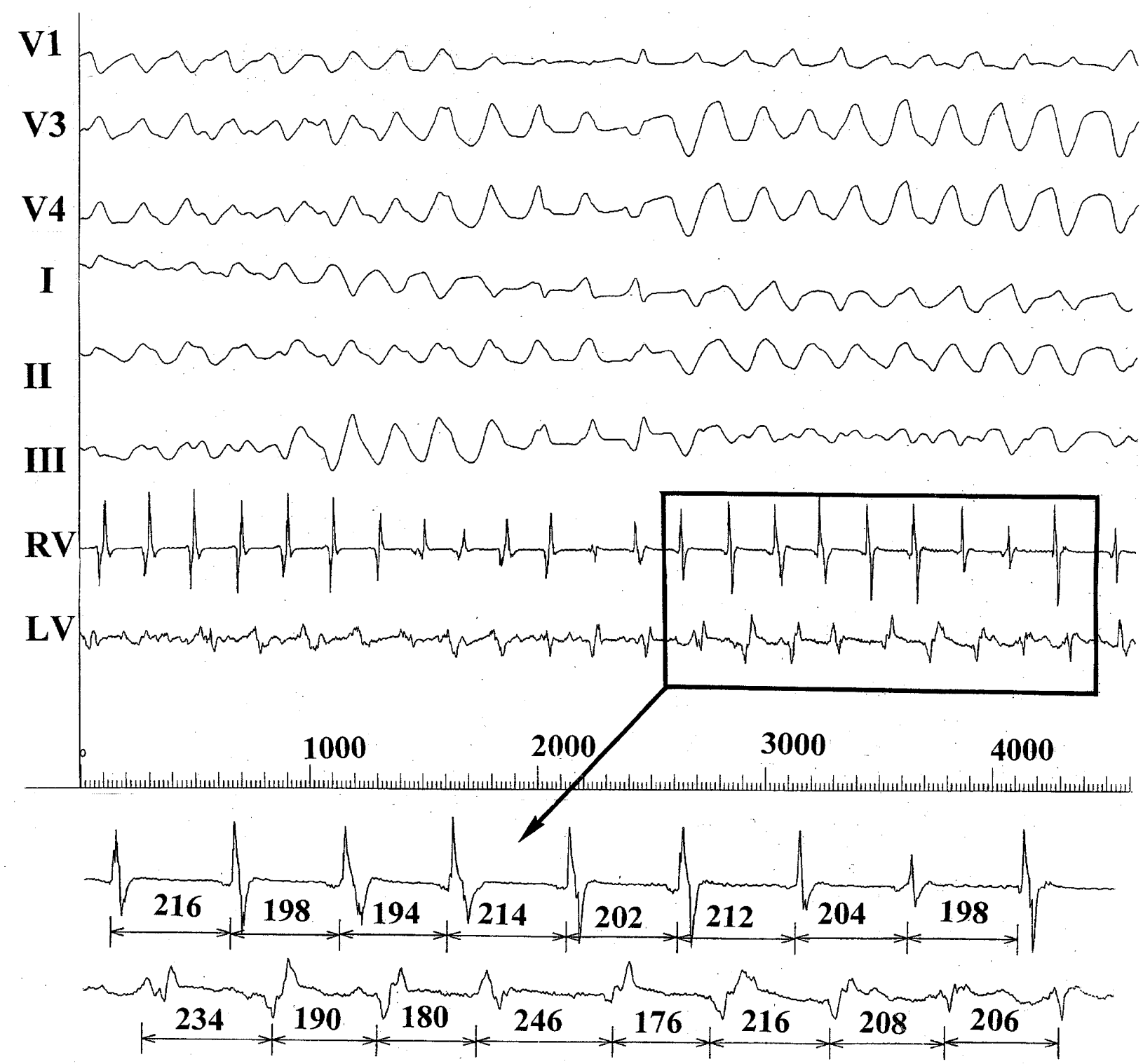

Figure I

Simultaneous recordings of the bipolar electrograms showing distinct differences of the local ventricular electrical activities between the right ventricular apex and the posterior left ventricle during an induced episode of ventricular fibrillation in a patient $(50 \mathrm{~mm} / \mathrm{sec})$. The ventricular fibrillation interval was shown in $\mathrm{ms}$. RV = right ventricle, $L V=$ left ventricle.

observed. The sharp and rapid deflections on the electrograms from the esophagus suggest they are mainly local electrical activities, although the amplitude of these electrograms are lower than those from the RV apex. This non- invasive approach for evaluating ventricular refractoriness and the dispersion of refractoriness between the RV and LV has not been described previously. Systematic findings 
Table I: The estimated VERP and its dispersion between the right ventricular apex and the posterior left ventricle during induced ventricular fibrillation.

\begin{tabular}{lccccc}
\hline & Mean & Median & 10th Percentile & Shortest & Variance \\
\hline RV apex & $186 \pm 25$ & $188 \pm 26$ & $146 \pm 40$ & $125 \pm 44$ & $2084 \pm 3914$ \\
Posterior LV & $185 \pm 17$ & $188 \pm 20$ & $145 \pm 26$ & $119 \pm 26$ & $1101 \pm 646$ \\
Dispersion & $13 \pm 9$ & $7 \pm 6$ & $40 \pm 27$ & $31 \pm 19$ & $620 \pm 595$ \\
\hline
\end{tabular}

Values are presented as mean \pm SD in ms. RV, right ventricular; LV, left ventricular.

with no complications in our study suggest the safety and feasibility of the method.

The mean, median, 10th percentile and the shortest VF intervals during VF were taken as estimates of the local VERP in this study. The differences of the estimated VERPs between the two sites were used as the dispersion of refractoriness between the RV apex and the posterior LV. The minimum values of the estimated VERPs in our study are similar with the mean VF interval that Ramdat Misier et al [9] measured in the noninfarcted area of the LV epicardium in patients with cardiac arrest and documented VF undergoing cardiac surgery $(129 \pm 3.4 \mathrm{~ms}]$. In our patients, the dispersion of the 10th percentile VF intervals between the RV apex and posterior LV was the largest among the dispersions of the estimated VERPs. Previous study suggest that the short fibrillation intervals might better estimate the atrial effective refractoty period during atrial fibrillation [16]. In this study, the dispersion of the 10th percentile and shortest VF intervals are approximately threefold of that of the mean and medium VF intervals. Since the shortest VF interval is more subjected to the influence of measurement errors, we are in the opinion that the 10th percentile VF interval may be a better estimate of the VERPs during VF.

The significance of disparity in refractoriness in the genesis of tachyarrhythmias has been emphasized by several experimental studies $[6,8,9]$. In the present study, the dispersion of the 10th percentile VF intervals between the RV apex and posterior LV is markedly larger than the previously reported dispersion of repolarization in patients without malignant ventricular arrhythmias $[14,15]$. It may suggest that inhomogeneous distribution of the ventricular refractoriness plays an important role in the initiation and/or perpetuation of $\mathrm{VF}$, presumably increasing the opportunity for reentry to occur. In this study, most patients had coronary heart disease. The diseased myocardial cell might be responsible for the increased dispersion of refractoriness [5].

\section{Study limitation}

In the present study, bipolar electrogram has been recorded at only one site each from the right and left ventricles. Recordings from multiple sites could probably provide more detailed information. Secondly, the previous studies in the canine ventricle $[6,13]$ suggested the process of fibrillation requires a minimum period of one minute before steady-state values are reached. For ethical considerations, the mean episodes of induced VF in our study were between 8.2 to 14.2 seconds. For the same reason, there is no data from control group patients without VF were obtained. However, our findings revealed nevertheless a markedly increased dispersion of ventricular refractoriness as compared with those without malignant ventricular arrhythmias in the previously published data $[14,15]$.

\section{Conclusion}

The present study verified the feasibility of recording local ventricular activities during induced VF via an esophageal electrode behind the posterior LV and via telemetry from the RV apex electrode of an implanted ICD and the usefulness of VF intervals obtained using this technique in evaluation of the dispersion of refractoriness in patients with ICD implantation. The dispersion of refractoriness between the RV apex and posterior LV was markedly greater than the previously reported dispersion of refractoriness in patients without malignant ventricular arrhythmias, suggesting the potential usefulness of this novel measurement in evaluation of the dispersion of refractoriness in patients with malignant ventricular arrhythmias.

\section{Competing interests}

None declared.

\section{Authors' contributions}

JL performed final data analysis and drafted the manuscript; CMP participated design of the study, clinical data collection and carried out part of the data analysis; $\mathrm{EH}$ participated in clinical data collection; OL carried out data collection and part of the data analysis; EL participated in clinical data collection; SB participated the design of the 
study, data analysis and the edit of the manuscript; SY conceived the design and coordinated the study, and performed part of the data analysis and participated in the writing of the manuscript. All authors read and approved the final manuscript.

\section{References}

I. Bardou AL, Auger PM, Achour S, Dumee P, Birkui PJ, Govaere MC: Effect of myocardial infarction and ischemia on induction of cardiac reentries and ventricular fibrillation. Acta Biotheor 1995, 43:363-72.

2. Kuo CS, Reddy CP, Muntakata K, Surawicz B: Mechanism of ventricular arrhythmias caused by increased dispersion. Eur Heart J 1 985, 6:63-70.

3. Kuo CS, Atarashi H, Reddy CP, Surawicz B: Dispersion of ventricular repolarisation and arrhythmia: study of two consecutive ventricular premature complexes. Circulation 1985, 72:370-6.

4. Lammers WJEP, Allessie MA, Rensma PL, Scalij MJ: The use of fibrillation cycle length to determine spatial dispersion in electrophysiological properties used to characterize the underlying mechanism of fibrillation. N Trends Arrhythmia 1986, 2: 109-I I2.

5. Misier AR, Opthof T, van Hemel NM, Defauw JJ, de Bakker JM, Janse $\mathrm{MJ}$, van Capelle FJ: Increased dispersion of "refractoriness" in patients with idiopathic paroxysmal atrial fibrillation. J Am Coll Cardiol 1992, 19:1531-5.

6. Opthof T, Misier AR, Coronel R, Vermeulen JT, Verberne HJ, Frank RG, Moulijn AC, van Capelle FJ, Janse MJ: Dispersion of refractoriness in canine ventricular myocardium: Effects of sympathetic stimulation. Circ Res 1991, 68:1204-1215.

7. Opthof T, Dekker LR, Coronel R, Vermeulen JT, van Capelle FJ, Janse $M J$ : Interaction of sympathetic and parasympathetic nervous system on ventricular refractoriness assessed by local fibrillation intervals in the canine heart. Cardiovasc Res 1993, 27:753-9.

8. Wang L, Li CY, Yong AC, Kilpatrick D: Fast Fourier transform analysis of ventricular fibrillation intervals to predict ventricular refractoriness and its spatial dispersion. PACE 1998 , 21:2588-95.

9. Misier AR, Opthof T, van Hemel NM, Vermeulen JT, de Bakker JM, Defauw |l, et al.: Dispersion of 'refractoriness' in noninfarcted myocardium of patients with ventricular tachycardia or ventricular fibrillation after myocardial infarction. Circulation 1995, 91:2566-72.

10. Roth JV, Brody JD, Denham EJ: Positioning the pacing esophageal stethoscope for transesophageal atrial pacing without P-wave recording: Implications for transesophageal ventricular pacing. Anesth Analg 1996, 83:48-54.

II. McEneaney DJ, Cochrane DJ, Anderson JA, Adgey AAJ: A gastroesophageal electrode for atrial and ventricular pacing. PACE 1997, 20:1815-25.

12. Santini M, Ansalone G, Cacciatore G, Turitto G: Transesophageal pacing. PACE 1990, 13:1298-1332.

13. Cha YM, Birgersdotter-Green U, Wolf PL, Peters BH, Chen PS: The mechanism of termination of reentrant activity in ventricular fibrillation. Circ Res 1994, 74:495-506.

14. Franz MR, Bargheer K, Rafflenbeul W, Haverich A, Lichtlen PR: Monophasic action potential mapping in human subjects with normal electrocardiograms: direct evidence for the genesis of the T wave. Circulation 1987, 75:379-386.

15. Shimizu W, Ohe T, Kurita T, Takaki H, Aihara N, Kamakura S: Early afterdepolarizations induced by isoproterenol in patients with congenital long QT syndrome. Circulation 1991, 84:1915-23.

16. Li Z, Hertervig E, Carlson J, Johansson C, Olsson SB, Yuan S: Dispersion of refractoriness in patients with paroxysmal atrial fibrillation. Evaluation with simultaneous endocardial recordings from both atria. J Electrocardiol 2002, 35:227-234.

\section{Pre-publication history}

The pre-publication history for this paper can be accessed here: http://www.biomedcentral.com/1471-2261/4/8/prepub
Publish with Biomed Central and every scientist can read your work free of charge

"BioMed Central will be the most significant development for disseminating the results of biomedical research in our lifetime. " Sir Paul Nurse, Cancer Research UK

Your research papers will be:

- available free of charge to the entire biomedical community

- peer reviewed and published immediately upon acceptance

- cited in PubMed and archived on PubMed Central

- yours - you keep the copyright

Submit your manuscript here:

http://www.biomedcentral.com/info/publishing_adv.asp
BioMedcentral 